\title{
El currículo oculto en la formación del médico. El caso de la subespecialidad en Cirugía Plástica, Estética y Reconstructiva
}

\author{
M. Candaudap-Ortega
}

Introducción. La formación médica es una necesidad pues serán los profesionales que resolverán nuestros problemas de salud. En 1994, el Consejo Universitario aprobó el Plan Único de Especializaciones Médicas (PUEM), pero desde los años treinta la subespecialidad en Cirugía Plástica, Estética y Reconstructiva fue introducida en los hospitales de salud pública. El PUEM ha establecido propósitos que son influidos por prácticas informales que están inmersas en cualquier proceso educativo, permitiendo aprendizajes reglamentados por el currículo oculto. Sujetos y métodos. Para saber cómo influyen las prácticas informales en la subespecialidad, se realizó un estudio descriptivo a un grupo de residentes de la especialidad. Resultados y conclusiones. Después de un año de observación de los residentes, charlas y una encuesta de nueve preguntas, conocimos algunas de las prácticas que se establecen más allá del currículo formal y el plan de estudios y que dan cuenta de las prácticas y acciones cotidianas que vive el médico, las cuales nada tienen que ver con lo establecido formalmente o de forma 'explicita'; sin embargo, aun estando ocultas o no contempladas en el currículo, dan cuenta también de la formación de los médicos. Los estudios de posgrado se ven afectados por los efectos de este currículo, que desempeña un papel importante en el desarrollo de los estudiantes y sus destinos, como son los recorridos educativos de las especializaciones médicas.

Palabras clave. Currículo. Educación informal. Formación. Función tutorial. Prácticas. Residente.

The hidden curriculum in medical training. The case of subspecialty in Plastic, Reconstructive and Aesthetic Surgery

Introduction. The medical education is a necessity because they are the professionals who will solve our problems. There- fore, the physician must choose a specialty, the study became more prominent postgraduate training in medicine. In 1994 the University Council approved the Single Plan for Medical Specializations (PUEM), but since the thirties the sub-specialty in Plastic Surgery, Cosmetic and Reconstructive was introduced in public health hospitals. The PUEM established purposes are influenced by informal practices that are engaged in any educational process, allowing learning regulated by the hidden curriculum. Subjects and methods. To know that influence the informal practices in the sub-specialty, an observational, prospective and descriptive of a group of residents in the specialty. Results and conclusions. After a year of observation of residents, talks and a survey of nine questions, they knew that the doctors asked to prepare a basis in education, as may best support. Therefore, you must create a curriculum that ensures the smooth function of the tutorial, to be of adequate guidance to residents in both the academic aspect as in the surgery, and thus benefit from the experience of physicians basis for their desire to serve Mexicans.

Key words. Curriculum. Informal education. Practice. Resident. Training. Tutorial role.

\section{Introducción}

La salud es indispensable para la vida; por ello se ha convertido en una preocupación constante la formación de médicos especialistas en ramas diversas, que puedan afrontar de manera ética y responsable los problemas de salud que aquejan a la sociedad.

La enseñanza de la medicina dentro de instituciones de salud, particularmente del Instituto Mexicano del Seguro Social, tiene poco más de 50
Facultad de Filosofía y Letras. Circuito Universitario. Universidad Nacional Autónoma de México. México DF, México.

\section{Correspondencia}

Michelle Candaudap Ortega. Facultad de Filosofía y Letras. Circuito Universitario. Universidad Nacional Autónoma de México. CP 04510. México DF, México.

E-mail

clear_pagdo@hotmail.com 
años, dentro de los cuales, la Universidad Nacional Autónoma de México (UNAM) ha avalado estos estudios. Cabe destacar que la especialización resulta ser el tipo de estudio formativo de posgrado más común dentro de la medicina [1].

En el año 1942 se organizó el Internado General Rotatorio, que incluía los servicios de las cuatro especialidades básicas (cirugía general, ginecoobstetricia, medicina interna y pediatría) en el Hospital General de México, a modo de complementar la formación de un médico general preparado para el ejercicio liberal o en condiciones de iniciar su formación de especialista en las instituciones. En 1994, el Consejo Universitario aprobó el Plan Único de Especializaciones Médicas (PUEM), organizado en una construcción conceptual funcional para conducir acciones educativas médicas, que establecieron diversos compromisos consensuados entre la Facultad de Medicina, las Instituciones de Salud y los Consejos Mexicanos de Especialidades Médicas.

La subespecialidad en Cirugía Plástica, Estética y Reconstructiva tiene sus inicios en los años treinta, con la práctica de intervenciones reconstructivas, pero sobre todo con los inicios de la cirugía estética. El Dr. Mario Gonzáles Ulloa introduce la práctica de la cirugía reconstructiva en los hospitales de salud pública fundando un servicio en el Hospital Universitario de Monterrey.

$\mathrm{Al}$ inicio de este posgrado, se adaptó al medio mexicano la experiencia obtenida de la residencia en los Estados Unidos [2], optándose por exigir como requisito para entrar una residencia de dos años en cirugía general, y solamente se recibían dos alumnos por año para cursar tres años más en cirugía plástica, estética y reconstructiva. Para obtener el reconocimiento universitario se aceptó el formato de los cursos de adiestramiento de la División de Estudios Superiores, inaugurándose así oficialmente la primera residencia auspiciada por la UNAM con un sistema de enseñanza tutelar, con participación directa del alumno en el estudio y tratamiento de los pacientes, siempre bajo la supervisión del personal médico de planta. Actualmente se cursan tres años en cirugía general y tres años más en cirugía plástica, estética y reconstructiva [1-3].

El PUEM ha establecido fines educativos, acorde con las necesidades específicas de salud de los mexicanos y las expectativas de la formación profesional de los estudiantes, pero Eduardo Acosta Arreguín menciona que siempre, al llevar a cabo un proceso educativo, encontraremos lo que se conoce como educación informal, la cual implica todos aquellos aprendizajes que suceden dentro del proceso formal, pero que no están explícitamente manifestados en los documentos referidos. Es con frecuencia más amplia e incluso puede tener una influencia determinante en el proceso educativo y su resultado final [3].

Este tipo de educación se da en cualquier nivel y cualquier institución; por lo tanto, la enseñanza de una subespecialidad médica no sería la excepción.

Desde la década de los ochenta, Jackson [4] señalaba que el currículo oculto tiene la capacidad de mostrar las contradicciones entre lo que se dice $y$ lo que se hace, por lo que resulta una exigencia que toda institución instruya permanentemente e informe de manera sistemática a los docentes sobre sus responsabilidades en tanto como intermediarios y facilitadores, a fin de no distorsionar los objetivos del currículo.

La sociología de la educación denomina 'currículo oculto' a aquellos aspectos no explícitos del currículo. Perrenoud [5] considera al respecto que este tipo de prácticas no son ningún secreto en realidad. De hecho, sabemos que el currículo oculto puede reforzar las desigualdades sociales existentes mediante la enseñanza de temas y comportamientos en función de la especialidad y estatus social de los estudiantes; de igual modo, puede favorecer las interacciones sociales de algunos estudiantes en el seno de los centros educativos, o bien convertirse en prácticas que lejos de ser formativas, son de trabajo no remunerado para los estudiantes $[3,6]$.

El currículo oculto, acaso más poderoso que el explícito, es el que da cuenta del juego entre las personalidades de los estudiantes a través de las interacciones cotidianas con profesores y personal de la institución, que luego quedan grabadas en la forma de valores y actitudes que delinean la 'manera de ser' [7].

Así, podemos preguntarnos cómo es el currículo oculto en la subespecialidad médica de Cirugía Plástica y Reconstructiva del Centro Médico La Raza.

\section{Sujetos y métodos}

Para saber cómo influyen las prácticas informales en la subespecialidad, se realizó un estudio 


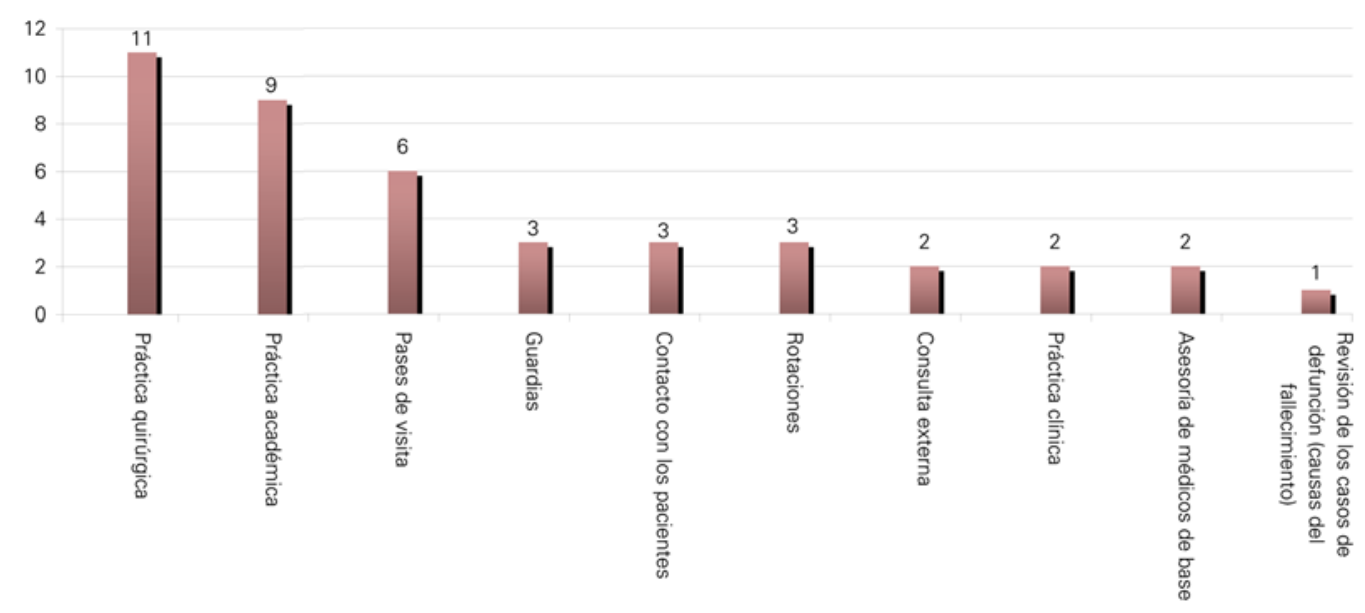

Figura 1. Pregunta 7: ¿qué prácticas benefician tu formación?

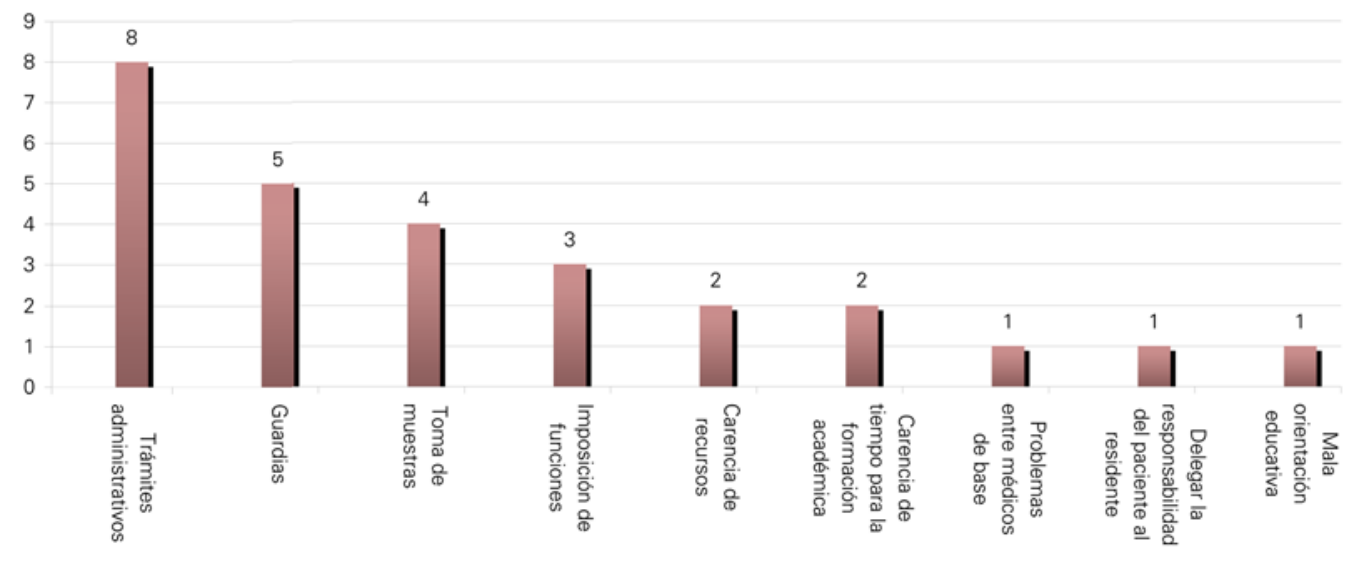

Figura 2. Pregunta 6: ¿qué prácticas no benefician tu formación?

observacional, definido como aquel en el que se presenciarán los fenómenos sin modificar intencionadamente las variables; prospectivo, porque se definirá previamente la forma de recolección de datos en los cuestionarios diseñados ex profeso; y descriptivo, ya que se estudiará a un solo grupo.

El grupo de estudio estuvo conformado por 9 residentes (tres de $4 .^{\circ}$ año, tres de $5 .^{\circ}$ año y tres de 6. ${ }^{\circ}$ año) y 3 licenciados del hospital de especialidades Centro Médico La Raza del Instituto Mexi- cano del Seguro Social, pertenecientes al Servicio de Cirugía Plástica y Reconstructiva.

Primeramente se realizó una observación durante cuatro meses de las actividades que se efectúan cotidianamente en el hospital; luego se mantuvieron charlas con los residentes para poder obtener una primera impresión del sentir que se tiene de su desarrollo como residentes.

Después se volvió a realizar una segunda observación mediante una encuesta de nueve preguntas, de las cuales cuatro fueron abiertas. 


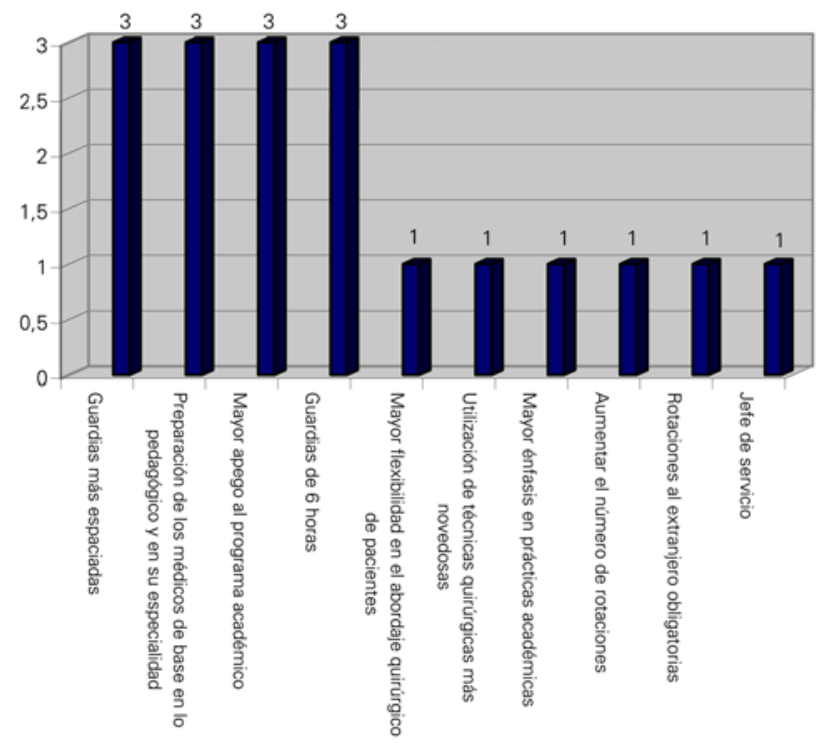

Figura 3. Pregunta 8: ¿qué cambiarías en la enseñanza de tu subespecialidad?

\section{Resultados}

Los residentes consideran que conocen las funciones tutoriales que deben realizar los médicos de base, pero menos de la mitad consideran que éstos las llevan a cabo (33\%). Por otra parte, el 92\% de los encuestados consideran que realizan actividades que no son parte de su formación como especialistas, pero dentro de las mismas hay prácticas que los benefician (Fig. 1). Complementando lo anterior, consideran que también existen prácticas que no benefician a su formación (Fig. 2).

Por último, mientras algunos estudiantes señalan que pedirían cambios en la subespecialidad para poder cumplir mejor con su desempeño académico (Fig. 3), otros preferirían que todo se mantuviera igual (Fig. 4).

\section{Discusión}

Se presume que los médicos residentes creen que la parte pedagógica está bien y sólo se necesitaría contar con médicos más preparados en esta área para desarrollarla de manera adecuada. Podría ser una buena solución que se contara con un de- partamento dentro del IMSS que tuviera apoyo de médicos especialistas y algunos pedagogos expertos en enseñanza médica que puedan conjuntar la parte teórica y práctica de la medicina con las cuestiones pedagógicas para lograr una correcta organización de las mismas, con lo cuál se lograría un desempeño académico del 100\%.

Las investigaciones que describen las situaciones vividas por los estudiantes tienen múltiples antecedentes en la bibliografía mundial, como los estudios de comprensión de los dilemas que influyen en su formación, además de su solución en la parte clínica.

Es importante mencionar que esta investigación surge de las opiniones de un reducido número de residentes de la subespecialidad en Cirugía Plástica, Estética y Reconstructiva; además, no corresponde a una investigación formal que nos dé resultados producto de un protocolo previamente diseñado para conocer las experiencias de todos los residentes. Sin embargo, constituye un indicador de lo que puede estar ocurriendo con el proceso de enseñanza-aprendizaje y su formación como especialistas.

Se han llevado a cabo investigaciones que analizan el currículo oculto en la formación ética de los médicos, la educación informal dentro de las 


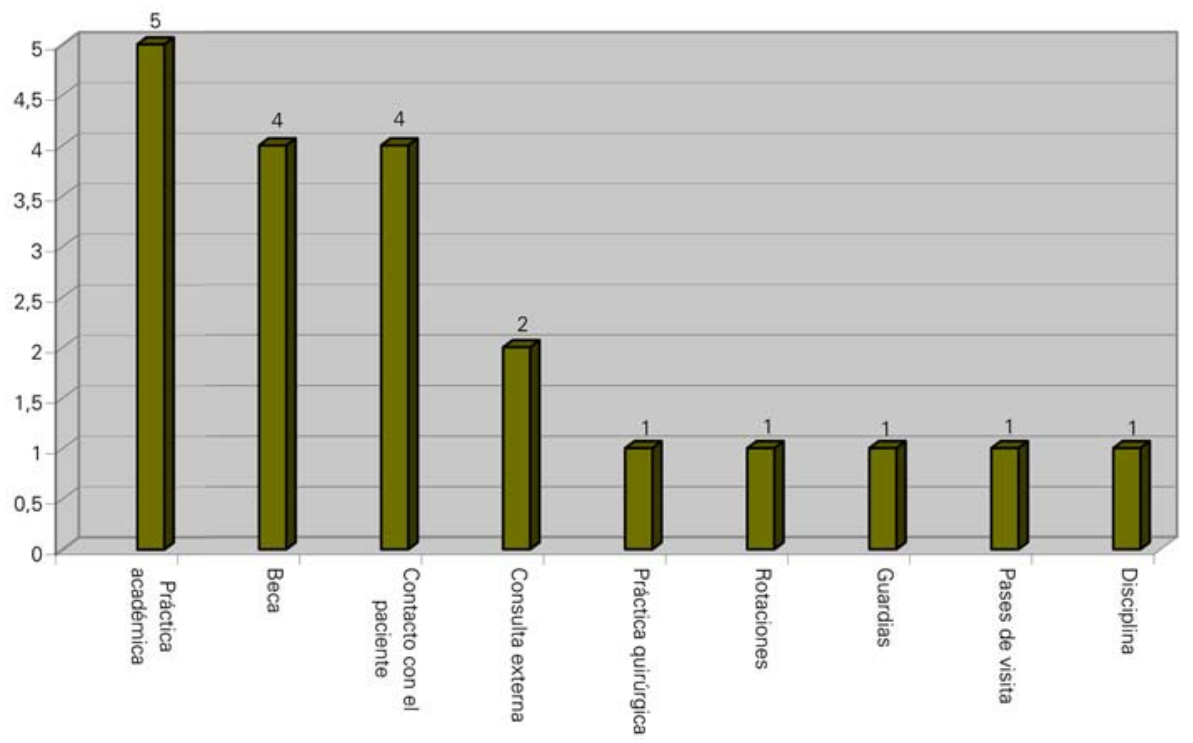

Figura 4. Pregunta 9: ¿qué no cambiarías en la enseñanza de tu subespecialidad?

residencias, y otros donde se menciona la parte tutorial dentro de la enseñanza médica.

Se requiere una base empírica para el buen desarrollo de la subespecialidad, la cual sólo puede obtenerse con la participación directa del residente que realiza la práctica clínica y quirúrgica.

\section{Bibliografía}

1. Solano LD. La enseñanza de la medicina. In Ruiz de Gauna-Bahíllo P, Solano-López D, Lafuente-Sánchez JV, eds. Enseñanza y medicina: mosaico de reflexiones en torno a la docencia de las ciencias médicas. Leioa: Servicio Editorial Universidad del País Vasco; 1999. p. 77-94.

2. De la Fuente JR. La educación médica y la salud en México: textos de debate. México: Siglo XXI; 1996.

3. Suárez F, Díaz E. La formación ética de los estudiantes de medicina: la brecha entre el currículo formal y el currículo oculto. Acta Bioética 2007. URL: http://www.scielo.cl/ scielo.php.pid.

4. Jackson P. La vida en las aulas. Madrid: Morata; 1991.

5. Perrenoud P. La construcción del éxito y del fracaso escolar. Madrid: Morata; 1990.

6. Leal FG. ¿Educando médicos? De Luis Echeverría Álvarez a Vicente Fox Quesada. México: Universidad Autónoma Metropolitana Unidad Xochimilco; 2007.

7. Colom CA. Continuidad y complementariedad entre la educación formal y la no formal. Revista de Educación 2005; 338: 9-22. 